Henning Timcke und Anita Nixon*

\title{
nanoo.tv - Die Schweizer Filmplattform und Online-Mediathek für Bildung
}

https://doi.org/10.1515/bfp-2020-2029

Zusammenfassung: Die Schweizer Filmplattform und Online-Mediathek für Bildung, nanoo.tv, ist seit 2006 in Schweizer Bildungsinstitutionen im Einsatz, von der Grundschule bis zur Tertiärstufe. Die Plattform ermöglicht, audiovisuelle Inhalte aus TV- und Radiosendern und anderen Quellen aufzunehmen, zu bearbeiten, und mit Lehrenden und Lernenden zu teilen. Zudem bietet die OnlineMediathek Zugriff auf über 300000 audiovisuelle Inhalte. ${ }^{1}$ Die Gründer von nanoo.tv haben maßgeblich zu den heute in der Schweiz geltenden urheberrechtlichen Voraussetzungen zur Nutzung von audiovisuellen Inhalten in der Bildung beigetragen. Aktuell wird die Medienplattform an über 140 Schweizer Bildungsinstitutionen eingesetzt. ${ }^{2}$

Schlüsselwörter: Mediathek; Filmplattform; Medienplattform; Videorekorder

\section{nanoo.tv - the Swiss Film Platform and Online Media Library for Education}

Abstract: The Swiss film platform and online media library for education, nanoo.tv, has been in use in Swiss educational institutions from primary school to tertiary level since 2006. The platform allows to record, edit, and share audio-visual content from TV programmes and other sources with teachers and students. Furthermore, the online media library allows access to more than 300,000 tutorials, feature films, documentaries and other audiovisual content. The founders of nanoo.tv have made a significant contribution to establishing the copyright conditions presently applicable to the use of audiovisual content in education in Switzerland. The media platform is currently used in over 140 Swiss educational institutions.

Keywords: Media library; film platform; media platform; video recorder

1 Stand September 2020.

2 Stand September 2020.

Henning Timcke, henning.timcke@nanoo.tv

*Kontaktperson: Anita Nixon, anita.nixon@nanoo.tv

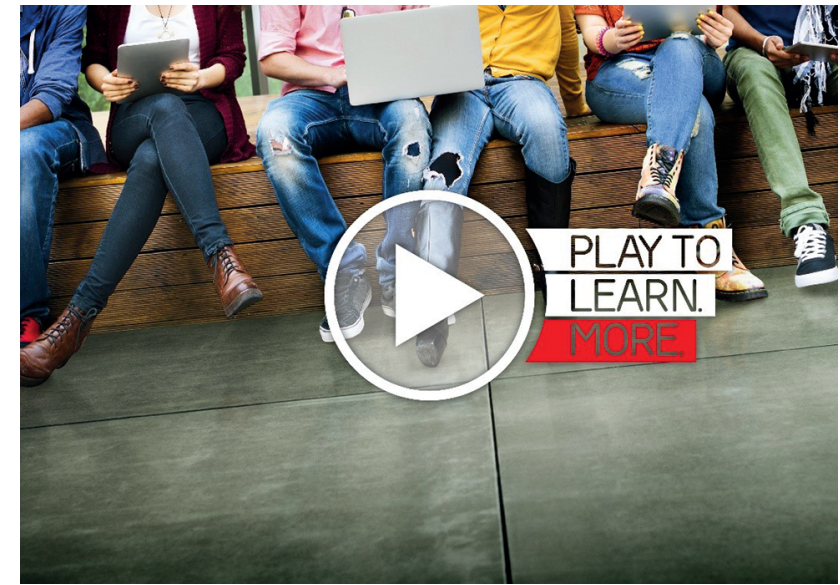

Abb. 1: Die Filmplattform und Online-Mediathek wird in der Schweiz an über 140 Bildungsinstitutionen eingesetzt

\section{Entstehung}

Der heute in nanoo.tv enthaltene Online-Videorecorder mit Replay-TV-Funktion wurde 1998 für das Schweizer Fernsehen entwickelt und kam bei den Olympischen Winterspielen in Salt Lake City zum Einsatz. Im Anschluss wurde nanoo.tv für den Einsatz in Bildungsinstitutionen weiterentwickelt und 2006 als Filmplattform an der Zürcher Hochschule der Künste (ZHdK) eingeführt. Die Hochschule hat bei der Bestimmung der gewünschten Features für Schulen wesentlich mitgewirkt.

Seit 2008 engagiert sich nanoo.tv für die Schaffung einer soliden urheberrechtlichen und organisatorischen Grundlage zur Verwendung von audiovisuellen Medien in der Bildung. So ist mit der Zeit eine klare Regelung für das Archivieren und das Vervielfältigen von TV-Filmen im Unterricht auf passwortgeschützten Plattformen wie nanoo.tv entstanden. ${ }^{3}$ In den Jahren 2014 und 2018 gewann nanoo. tv den Worlddidac Award. ${ }^{4}$ Der Preis wird seit dreißig Jahren von der Worlddidac Stiftung an Anbieter von innovativen Bildungsprodukten vergeben. Die Jury hat neben

3 siehe Gemeinsamer Tarif 9 III, seit 2017 Gemeinsamer Tarif 7. 4 https://worlddidacaward.org/review2018, https://worlddidac.org/ wp-content/uploads/2017/05/Award-Winners-Brochure-2014.pdf. 
den technischen Funktionen insbesondere den Verdienst einer legalen Nutzung von audiovisuellen Medien in der Bildung hervorgehoben.

Die Betreiberin von nanoo.tv ist nahe Zürich in Baden angesiedelt und betreibt und steuert das rasant wachsende Filmarchiv im Medienzentrum der ZHdK in Zürich und beim Schweizer Telekomunternehmen Swisscom in Olten, wo ebenfalls die Signale der TV-Programme empfangen werden. Als Mitinitiantin und Produzentin der Bildungstagung PLAY TO LEARN ${ }^{5}$ engagiert sich das Team von nanoo.tv auch auf inhaltlicher Ebene für eine lernfördernde und kreative Verwendung von audiovisuellen Medien in der Bildung.

\section{Verbreitung und Nutzung}

\subsection{Schulen}

Die Filmplattform nanoo.tv wird zurzeit an über 140 Schweizer Bildungsinstitutionen aller Stufen genutzt: an Grundschulen, an der Sekundarstufe I, der Sekundarstufe II (sowohl an allgemeinbildenden wie auch an berufsbildenden Schulen) sowie an Hochschulen. Die zurzeit bestehende User-Community beläuft sich auf über 35000 Lehrkräfte, ${ }^{6}$ etwas mehr als die Anzahl Studierenden der Technischen Universität Berlin. ${ }^{7}$ Die User sind hauptsächlich Lehrpersonen und Dozierende, aber auch Bibliotheksmitarbeitende und weitere wissenschaftliche Mitarbeitende. Aktuell wird die Filmplattform in 19 Schweizer Kantonen eingesetzt. Eine Nutzung außerhalb der Schweiz ist in Planung; nanoo.tv engagiert sich für die Erarbeitung einer rechtlichen Grundlage für die urheberrechtliche Abgeltung.

\subsection{Hochschulbibliotheken}

\subsubsection{Medien- und Informationszentrum der Zürcher Hochschule der Künste}

Wie eingangs erwähnt, wurde die Filmplattform nanoo. tv in Zusammenarbeit mit der Zürcher Hochschule der Künste entwickelt. Heute bietet das Medien- und Informationszentrum (MIZ) der Zürcher Hochschule der Künste

5 https://www.play-to-learn.ch/.

6 Stand September 2020.

7 https://www.tu.berlin/ueber-die-tu-berlin/profil/tu-berlin-in-zah len/. sein Archiv mit nanoo.tv für die Tertiärstufe über den Nebis ${ }^{8}$-Katalog als Video-Streams an. ${ }^{9}$ In Nebis haben sich 140 Bibliotheken von Hochschulen, Fachhochschulen und Forschungsanstalten sowie Spezialbibliotheken aus allen Sprachregionen zusammengeschlossen. Der Zugriff erfolgt über SWITCH edu-ID. ${ }^{10}$ Das MIZ-Online-Archiv, das vom MIZ-Team seit den 1980er-Jahren ${ }^{11}$ aufgebaut und kuratiert wird, enthält bereits über 18000 Mediendateien. ${ }^{12}$

\subsubsection{Weitere (Hochschul-)Bibliotheken}

Mit weiteren Bibliotheken ist nanoo.tv im Gespräch, zum Beispiel mit der Bibliothek der Universität St. Gallen (HSG), mit der Bibliothek der Pädagogischen Hochschule Zürich (PHZH), mit der Bibliothek der Fachhochschule Nordwestschweiz (FHNW) sowie der Bibliothek der Hochschule Luzern (HSLU). An der Zentralbibliothek Zürich laufen zurzeit Pilotprojekte mit den Produkten nanoo.tv und nanoo.id.

\section{Nachfrage seit Ausbruch Covid-19}

Der Ausbruch der Covid-19-Pandemie wirkte sich allgemein stark auf die Nachfrage nach Video-Streaming aus; auch bei nanoo.tv zeigte sich dieser vermehrte Bedarf.

So verzeichnete die Filmplattform seit dem Ausbruch der Covid-19-Pandemie eine markante Zunahme der Nutzung von Video-Streaming durch bestehende Kunden: Im zweiten Quartal 2020 wurde gegenüber dem zweiten Quartal 2019 eine Zunahme der Nutzung um über 350 \% festgestellt.

Hinzu kam eine überdurchschnittliche Zunahme an neuen Kunden. Ab August bis heute verzeichnete nanoo.tv eine Zunahme von über 1600 neuen Nutzenden. ${ }^{13}$ Allen interessierten Bildungsinstitutionen stellte nanoo.tv ab Start des Lockdowns im März bis Ende Juli eine reduzierte Version der Filmplattform kostenlos zur Verfügung.

8 Nebis steht für das Netzwerk von Bibliotheken und Informationsstellen in der Schweiz.

9 https://www.zhdk.ch/miz/miz-nanootv.

10 switch.ch/edu-id.

11 Putschert (2009) 41.

12 https://www.zhdk.ch/miz/miz-nanootv.

13 Stand September 2020. 


\section{Funktionen}

\subsection{Verbindung von Autorisierungs- und Inhaltsdiensten}

Die Filmplattform nanoo.tv nutzt den Dienst nanoo.id, um Autorisierungs- und Inhaltsdienste zu verbinden. Er ermöglicht das einfache Verbinden und Anbinden von unterschiedlichen Autorisierungs- und Inhaltsdiensten. Zudem unterstützt der Dienst eine Vielzahl an zentralen Anmeldesystemen, beispielsweise Microsoft 365 oder Switch AAI. So erhält nanoo.id die Informationen über Zugangsberechtigungen und verbindet sie mit den entsprechenden Diensten, die Inhalte zur Verfügung stellen (beispielsweise Verlage). Die Nutzenden müssen sich nur einmal in ihrem zentralen Login-System anmelden. Die Zugangsberechtigungen sind bereits hinterlegt und müssen nicht erneut angegeben werden.

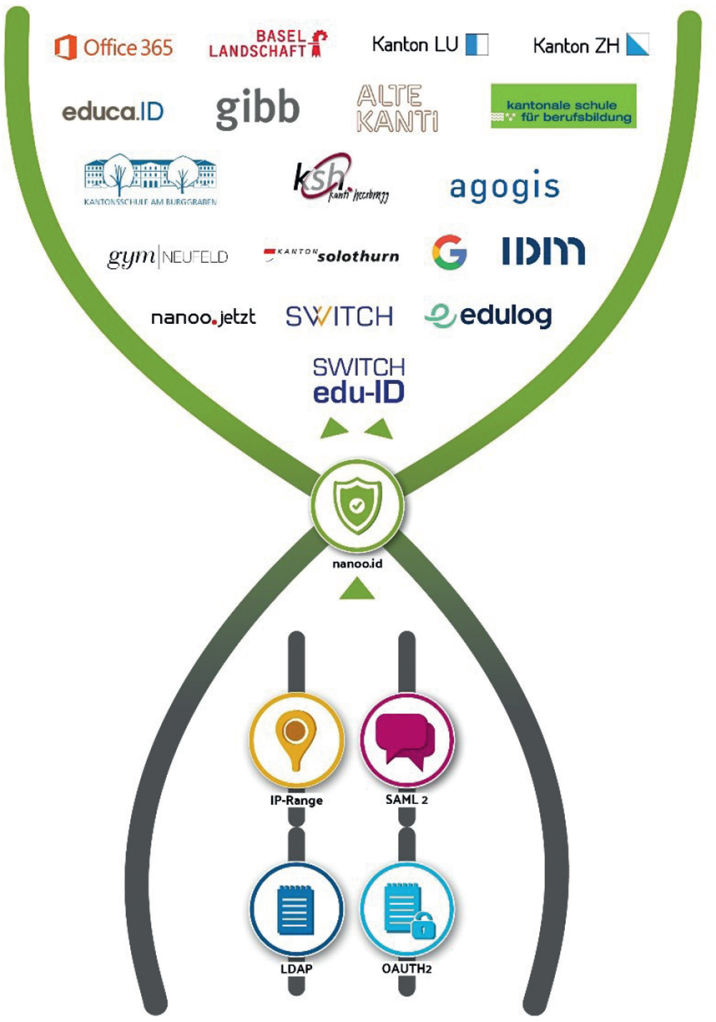

Abb. 2: Die Filmplattform nanoo.tv nutzt den Dienst nanoo.id, um Autorisierungs- und Inhaltsdienste zu verbinden

\subsection{Weitere zentrale Funktionen}

Nebst dem Verbinden von Autorisierungs- und Inhaltsdiensten ermöglicht die Plattform den Nutzern das Su- chen, Aufnehmen, Bearbeiten, Speichern, Kuratieren und Teilen von audiovisuellen Inhalten. Die Funktionen beinhalten:

- Suche über die gesamte Online-Mediathek aller teilnehmenden Bildungsinstitutionen

- Aufnahme von insgesamt 82 TV- und Radiosendern aus der Schweiz und aus Deutschland, Frankreich, Italien, Österreich, Spanien und England (z.B. 3sat, Arte, $\mathrm{BBC}, \mathrm{CNN})^{14}$

- Aufnahme von vergangenen und zur Aufnahme vormerken von zukünftigen TV- und Radiosendungen. Die Aufnahmen werden von den Nutzenden vorgenommen. (Lehrkräfte, Dozierende, Mitarbeitende Mediatheken)

- Aufbau von gemeinsamen Filmsammlungen in Fachbereichen, für die gesamte Organisation und organisationsübergreifend

- Vergabe von Rollen für die Organisation von Filmsammlungen und Administratorenrechte

- Empfehlungsfunktionen mittels gesicherter Hyperlinks zur Erhöhung der Qualität von gemeinsamen Filmsammlungen

- Zugriff auf spezialisierte Fach-Filmsammlungen (siehe Abschnitt „Inhalte“)

- Teilen von Filmen via Zugangslinks und mittels automatisch erzeugten $\mathrm{QR}$-Codes

- Unlimitierter Speicherplatz

- Zur schnellen Aufnahme bereitgestellte häufig genutzte und wiederkehrende aktuelle Sendungen (z.B. Nachrichten oder Sternstunde Philosophie)

- Hinzufügen von technischen und bibliografischen Metadaten und Katalogisaten sowie Unterrichtsunterlagen zu den Mediendateien

- Integriertes Werkzeug zum Schneiden von bestimmten Ausschnitten oder zum Wegschneiden von Werbung

- Durchführung von Videokonferenzen mit dem integrierten Open-Source-Webkonferenzsystem Big Blue Button

- Aufzeichnung von Videokonferenzen und automatisches Hochladen in das persönliche Archiv

- Direkte Videoaufnahme und automatisches Hochladen in das persönliche Archiv

- Direkte Bildschirmaufnahme (beispielsweise für Erklärvideos) und automatisches Hochladen in das persönliche Archiv

- Teilen der Videokonferenz-Aufzeichnungen und Video- und Bildschirmaufnahmen via Zugangslinks

14 Stand September 2020 


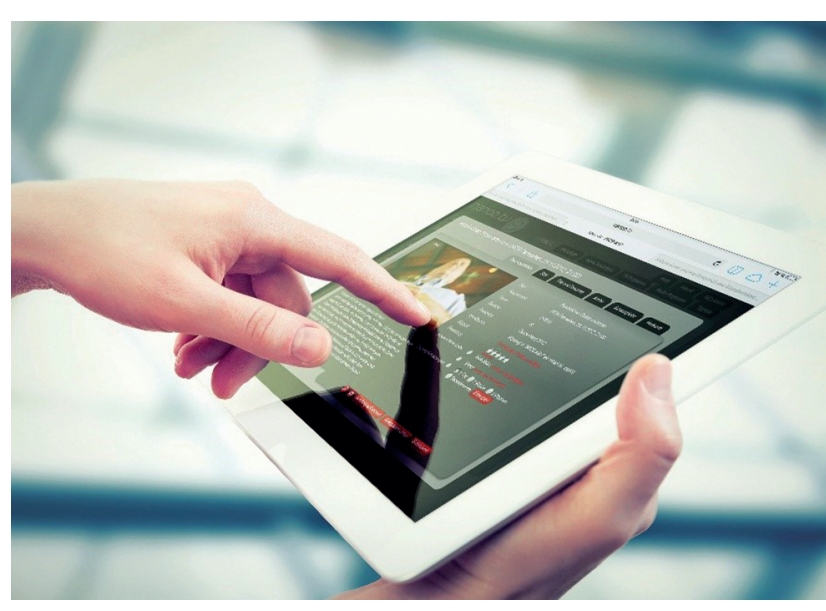

Abb. 3: Die Filmplattform ermöglicht Nutzern das Aufnehmen, Bearbeiten, Speichern, Kuratieren und Teilen von audiovisuellen Inhalten sowie den Zugriff auf die gesamte Online-Mediathek

\section{Inhalte}

Die Mehrheit der Inhalte wird von den Nutzenden aus den Bildungsinstitutionen beigesteuert. Somit bilden die Inhalte größtenteils die für den Bildungsauftrag an Grundschulen, Sekundarschulen sowie der Tertiärstufe nötigen Inhalte $\mathrm{ab}$.

Um zusätzliche Mediatheken für die Nutzenden zur Verfügung zu stellen, hat nanoo.tv vertragliche Regelungen mit Inhaltsanbietern erarbeitet. So stehen heute die Filmsammlungen der Stiftung Kinokultur ${ }^{15}$ und ausgesuchte Sammlungen des Bildungsanbieters der Schweizer Nationalbank, iconomix ${ }^{16}$ für die berechtigten Nutzer bereit.

Ebenfalls bestehen Verträge mit Verlagshäusern, beispielsweise mit der deutschen Firma GIDA. ${ }^{17}$ Kunden des Verlagshauses GIDA können ihre gekauften Medien statt über eine separate Plattform direkt in nanoo.tv abrufen. Somit sind alle Mediendateien für die Kunden über eine zentrale Plattform nutzbar. Mit weiteren Verlagshäusern sind Gespräche im Gang, z. B. mit dem Anbieter FWU, dem Medieninstitut der deutschen Bundesländer. ${ }^{18}$

15 www.kinokultur.ch.

16 www.iconomix.ch.

17 www.gida.de.

18 www.fwu.de.

\subsection{Digitalisierung von DVD-Sammlungen}

Sowohl an Schulen wie auch in den (Hochschul-)Bibliotheken wird die Möglichkeit der Digitalisierung von bestehenden DVD-Sammlungen dringlicher. Immer mehr Kunden gelangen mit diesen Anfragen an nanoo.tv. Die DVD-Bestände von Bildungsinstitutionen wie Schulen oder (Hochschul-)Bibliotheken sind oftmals ähnlich. Anstatt dass jede Institution ihre Bestände separat digitalisiert, können gemeinsame Sammlungen angelegt und gemeinsame Video-Streaming-Dienste lizenziert werden. Die Filmplattform nanoo.tv bietet dazu die technologische Infrastruktur. Allerdings müssen für solche Projekte die Abgeltungen neu geregelt werden. Deshalb ist nanoo.tv bestrebt, mit den beteiligten Akteuren zukunftsweisende Lösungen zu erarbeiten.

\section{Die rechtlichen Grundlagen in der Schweiz}

In aller Kürze: Das Schweizerische Urheberrecht erlaubt ,jede Werkverwendung der Lehrperson für den Unterricht in der Klasse“ (Art. 19 Abs. 1 lit. b URG), ${ }^{19}$ sowie „das Vervielfältigen von Werkexemplaren in Betrieben, öffentlichen Verwaltungen, Instituten, Kommissionen und ähnliche Einrichtungen für die interne Information und Dokumentation“ (Art 19 Abs. 1 lit. c URG). Diese Verwendungen sind verwertungsgesellschaftenpflichtig (Art 20 Abs. 2 und 4 URG).

Kopien von Fernsehsendungen sind für diese Bereiche zulässig, aber auch vergütungspflichtig. Dies wird im sogenannten Gemeinsamen Tarif 7 (Schulische Nutzung) sowie 9-I-VI geregelt. Der GT 7 schließt auch schulinterne Mediatheken sowie regionale oder kantonale Medienstellen als berechtigte Nutzer mit ein. Zudem erlaubt der Tarif die Vervielfältigung ganzer Radio- und TV-Sendungen und das Zugänglichmachen für berechtigte Nutzer auf passwort-geschützten digitalen Plattformen. ${ }^{20}$ Diese Nutzung wird zusätzlich an die Verwertungsgesellschaften vergütet. Die Lösung ermöglicht den Bildungsinstitutionen „von Angeboten wie nanoo.tv zu profitieren“. ${ }^{21}$

19 Bundesgesetz über das Urheberrecht und verwandte Schutzrechte (Urheberrechtsgesetz, URG) vom 9. Oktober 1992 (Stand 1.4.2020). Die Bundesversammlung der Schweizerischen Eidgenossenschaft. http s://www.admin.ch/opc/de/classified-compilation/19920251/.

20 https://prolitteris.ch/fileadmin/user_upload/ProLitteris/Dokume nte/Tarife_ab_2017/GT_7_Merkblatt_2017_2021.pdf.

21 Meier (2014) 96. 
„Dank technischer Innovation entstandene neue Geschäftsmodelle auch rechtlich $\mathrm{zu}$ ermöglichen und den Berechtigten im Gegenzug eine angemessene Vergütung für solche neuen Nutzungen zu sichern: dies ist eine zentrale Leitlinie einer idealen Verwertungsgesellschaft"..22

\section{Literaturverzeichnis}

Meier, Dieter (2014): Zur Verbreitung audiovisueller Werke in einer digitalen Schweiz. In: Wege zum idealen Verwertungssystem. Liber amicorum für Ernst Hefti, hg. v. Mathis Berger und Werner Stauffacher, 87-104. Basel: Helbing Lichtenhahn.

Putschert, Peter (2009): digitale schatzkammern. In: Zett - Das Magazin der Zürcher Hochschule der Künste, 2 (9), 41.

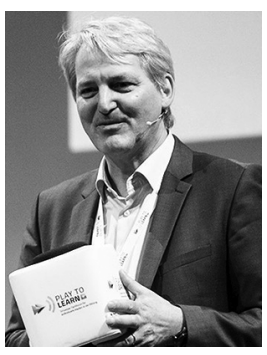

Henning Timcke

CEO nanoo.tv

Landstrasse 1

CH-5415 Rieden bei Baden

Schweiz

henning.timcke@nanoo.tv

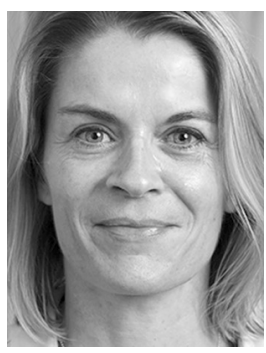

\section{Anita Nixon}

Öffentlichkeitsarbeit nanoo.tv

Landstrasse 1

CH-5415 Rieden bei Baden

Schweiz

anita.nixon@nanoo.tv

22 Meier (2014) 97. 